\title{
Hyperelliptic Integrals and Multiple Hypergeometric Series
}

\section{By Jean-Francis Loiseau, Jean-Pierre Codaccioni, and Régis Caboz}

Abstract. We consider the complete hyperelliptic integral

$$
J(a)=\int_{\alpha(a)}^{\beta(a)} \frac{d x}{\sqrt{a-P_{n}(x)}},
$$

where $a>0$ and

$$
P_{n}(x)=\sum_{k=2}^{n} \lambda_{k} x^{k},
$$

with $\lambda_{2}>0,[\alpha, \beta]$ being the connected component of $\left\{x \mid P_{n}(x) \leq a\right\}$ containing the origin.

Using a recent result concerning the Taylor expansion of the $\delta$-Dirac function, we write $J(a)$ as a power series of a parameter involving $a$ and the $\lambda_{k}$ 's.

We prove this series to be a sum of multiple hypergeometric series which reduces to a single term when the number of odd monomial terms in $P_{n}$ is less than or equal to one.

The region of convergence is then studied and a few particular cases are detailed.

1. Introduction. Of fundamental importance in mechanics is the integral

$$
t=\int_{q_{0}}^{q} \frac{d x}{\sqrt{2(E-U(x))}}
$$

which represents for a one-dimensional conservative mechanical system the time necessary to go from the initial point $q_{0}$ to the current point on the $q$-axis, $U(x)$ being the potential and $E$ the total energy.

Of particular interest is the case where $U(0)=0$ and $d U(0) / d x=0$, i.e., $U(x)=\frac{1}{2} \omega_{0}^{2} x^{2}+U_{1}(x)$, which exhibits a harmonic (quadratic) term together with an anharmonic (superquadratic) part $U_{1}(x)$. This anharmonic potential may be modeled by a polynomial.

The "complete" integral

$$
T(E)=2 \int_{q_{\min }}^{q_{\max }} \frac{d x}{\sqrt{2(E-U(x))}}
$$

represents the period of the oscillations when the movement is bounded, $q_{\min }$ and $q_{\max }$ being functions of the energy $E$.

In this paper we shall be dealing with the complete integral

$$
J(a)=\int_{\alpha(a)}^{\beta(a)} \frac{d x}{\sqrt{a-P_{n}(x)}},
$$

Received February 2, 1987; revised July 15, 1987.

1980 Mathematics Subject Classification (1985 Revision). Primary 33A25, 33A35. 


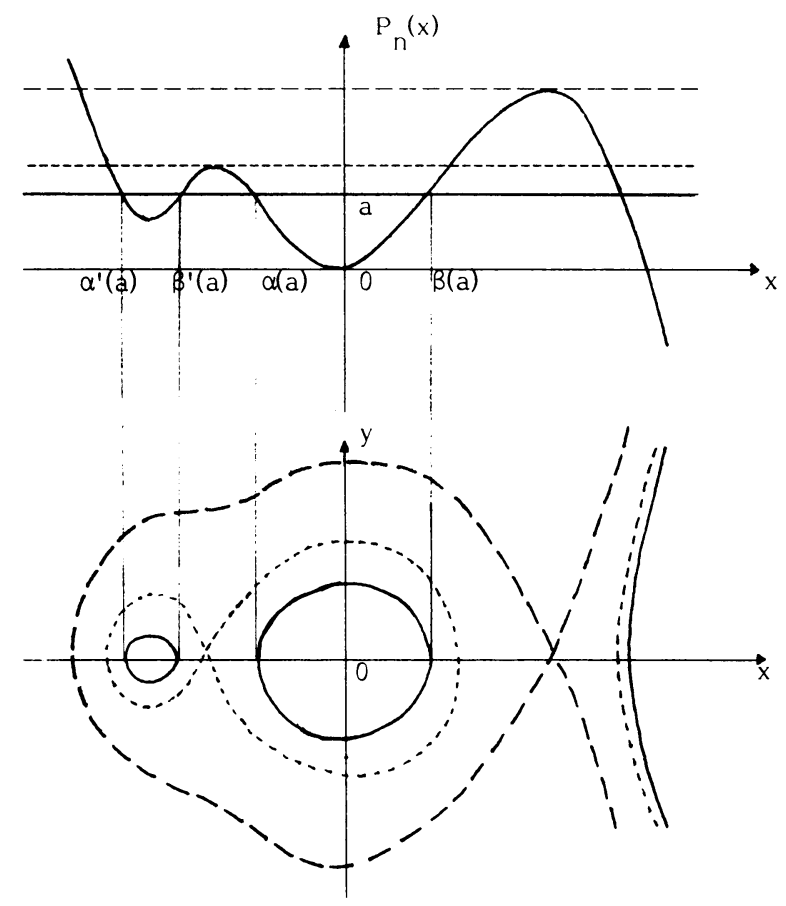

FIGURE 1

A typical case for $P_{n}(x)$.

(Separatrices are indicated by dotted lines)

where $a>0 . P_{n}(x)$ is a polynomial of degree $n, P_{n}(x)=\sum_{k=2}^{n} \lambda_{k} x^{k}$ with $\lambda_{2}>0$, and $[\alpha, \beta]$ the connected component of $\left\{x \mid P_{n}(x) \leq a\right\}$ containing the origin.

According to Carlson's definition [3], this integral is hyperelliptic as soon as $n>4$. A few cases may be reduced by a suitable change of variable to complete elliptic integrals [10], [12].

To show the link with multiple hypergeometric series, we shall use a property of the Dirac-distribution on a smooth manifold, recently proved [1] and already used in the simple case $P_{n}(x)=\lambda_{2} x^{2}+\lambda_{n} x^{n}[12]$.

Note that in this particular case, for $n=3,4$ or $6, J(a)$ can be directly integrated, even in the incomplete case (for a systematic review and a few references, see [12]). For the general case $P_{n}(x)$, the incomplete case must be treated in a different way, which will be examined in a forthcoming paper. The present study is therefore devoted to the complete integral.

2. Computation of $J(a)$. Setting $y^{2}(x, a)=a-P_{n}(x)$, the integral takes the form

$$
J(a)=\int_{\alpha(a)}^{\beta(a)} \frac{d x}{y(x, a)} .
$$

In the neighborhood of the origin, $P_{n}(x)$ stays close to $\lambda_{2} x^{2}$, and since $\lambda_{2}>0$, there exists an interval containing the origin where $P_{n}(x)>0$.

A typical case is depicted in Figure 1 where, for the chosen value $a$, the curve $y^{2}=a-P_{n}(x)$ has two bounded connected components and one unbounded. 
We only deal with the $[\alpha(a), \beta(a)]$ interval of integration, but the following method could be used for $\left[\alpha^{\prime}(a), \beta^{\prime}(a)\right]$, provided we perform a translation of axis, setting the local minimum of $P_{n}(x)$ as the new origin.

Since $d y / d a=1 / 2 y(x, a), J(a)$ may be written as

$$
J(a)=2 \int_{\alpha(a)}^{\beta(a)} \frac{d y}{d a} d x
$$

and since $y(\alpha, a)=y(\beta, a)=0$, differentiation and integration commute, so that

$$
J(a)=2 \frac{d}{d a} \int_{\alpha(a)}^{\beta(a)} y(x, a) d x=\frac{d}{d a} \int_{\alpha(a)}^{\beta(a)} d x \int_{-y(x, a)}^{+y(x, a)} d y .
$$

If we denote by $D_{a}$ the compact connected component containing the origin of the domain, defined by $z(x, y) \equiv y^{2}+P_{n}(x) \leq a$, we can write

$$
J(a)=\frac{d}{d a} \iint_{D_{a}} d x d y .^{*}
$$

Using the two-dimensional Heaviside characteristic function of the manifold

$$
\theta(z-a)= \begin{cases}1 & \text { if } z \leq a \\ 0 & \text { if } z>a\end{cases}
$$

the distribution $\delta(z-a)$ and its successive derivatives are defined by [8]

$$
\left\{\begin{array}{l}
\delta(z-a)=\frac{d}{d a} \theta(z-a), \\
\delta^{(k)}(z-a)=(-1)^{k} \frac{d^{k}}{d a^{k}} \delta(z-a), \quad k=1,2, \ldots
\end{array}\right.
$$

We may then write

$$
J(a)=\iint \delta(z-a) d x d y
$$

(integration being performed on the whole $(x, y)$ space). Setting now $z(x, y)=$ $z_{0}(x, y)+z_{1}(x)$, with

$$
\left\{\begin{array}{l}
z_{0}(x, y)=y^{2}+\lambda_{2} x^{2} \\
z_{1}(x)=\sum_{k=3}^{n} \lambda_{k} x^{k}
\end{array}\right.
$$

a result by Caboz, Codaccioni and Constantinescu [1] shows that for every value of $a$ for which there exists a compact subset of $\mathbf{R}^{2}$ containing the $\left\{z_{0}(x, y)=a\right\}$ manifold and a compact component of the $\{z(x, y)=a\}$ manifold with no critical point $(\overrightarrow{\operatorname{grad}} z \neq \overrightarrow{0})$, we can write

$$
\delta(z-a)=\sum_{k=0}^{\infty} \frac{1}{k !} z_{1}^{k} \delta^{(k)}\left(z_{0}-a\right) .
$$

(Here, the condition $\overrightarrow{\operatorname{grad}} z \neq \overrightarrow{0}$ excludes any separatrix.)

* Note that from a mechanical point of view, and aside from a scale factor, the double integral is nothing but the so-called action-integral taken over one cycle of the oscillation in time in the theory of action-angle variables (see, e.g., H. Goldstein, Classical Mechanics, Addison-Wesley, 1980). 
The change of coordinates

$$
\left\{\begin{array}{l}
x=\sqrt{\frac{z_{0}}{\lambda_{2}}} \cos \varphi \\
y=\sqrt{z_{0}} \sin \varphi
\end{array}\right.
$$

with Jacobian $1 / 2 \sqrt{\lambda_{2}}$, applied in (6), yields

$$
\begin{aligned}
J(a)=\sum_{k=0}^{\infty} \frac{1}{k !} \iint\left[\lambda_{3}\left(\frac{z_{0}}{\lambda_{2}}\right)^{3 / 2} \cos ^{3} \varphi+\cdots+\lambda_{n}\left(\frac{z_{0}}{\lambda_{2}}\right)^{n / 2} \cos ^{n} \varphi\right]^{k} & \\
& \times \delta^{(k)}\left(z_{0}-a\right) \frac{d z_{0} d \varphi}{2 \sqrt{\lambda_{2}}}
\end{aligned}
$$

and using the generalized binomial formula [13], we get

$$
\begin{gathered}
J(a)=\frac{1}{2 \sqrt{\lambda_{2}}} \sum_{\substack{k=0 \\
\sum_{3}, \ldots, k_{n} \\
\rho_{n}=k}}^{\infty} \frac{\lambda_{3}^{k_{3}} \cdots \lambda_{n}^{k_{n}}}{k_{3} ! \cdots k_{n} !} \int\left(\frac{z_{0}}{\lambda_{2}}\right)^{\sigma_{n} / 2} \delta^{(k)}\left(z_{0}-a\right) d z_{0} \\
\times \int_{0}^{2 \pi} \cos ^{\sigma_{n}} \varphi d \varphi
\end{gathered}
$$

where

$$
\left\{\begin{array}{l}
\rho_{n}=k_{3}+k_{4}+\cdots+k_{n} \\
\sigma_{n}=3 k_{3}+4 k_{4}+\cdots+n k_{n}
\end{array}\right.
$$

We shall use in the following also

$$
\tau_{n}=\sigma_{n}-2 \rho_{n}=k_{3}+2 k_{4}+\cdots+(n-2) k_{n},
$$

which has the same parity as $\sigma_{n}$. From the classical results [10]

$$
\int_{0}^{2 \pi} \cos ^{\sigma_{n}} \varphi d \varphi= \begin{cases}0 & \text { if } \sigma_{n} \text { is odd } \\ \frac{2 \sqrt{\pi} \Gamma\left(\sigma_{n} / 2+1 / 2\right)}{\left(\sigma_{n} / 2\right) !} & \text { if } \sigma_{n} \text { is even }\end{cases}
$$

( $\Gamma$ being the Gamma function) and

$$
\int\left(\frac{z_{0}}{\lambda_{2}}\right)^{\sigma_{n} / 2} \delta^{(k)}\left(z_{0}-a\right) d z_{0}=(-1)^{k} \frac{\left(\sigma_{n} / 2\right) !}{\left(\sigma_{n} / 2-k\right) !} \frac{a^{\sigma_{n} / 2-k}}{\lambda_{2}^{\sigma_{n} / 2}}
$$

we deduce

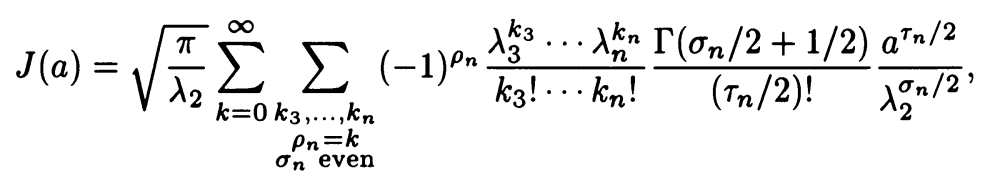

and setting

$$
A_{k_{3}, \ldots, k_{n}} \equiv \begin{cases}0 & \text { if } \sigma_{n} \text { is odd } \\ \frac{1}{k_{3} ! \cdots k_{n} !} \frac{\Gamma\left(\sigma_{n} / 2+1 / 2\right)}{\left(\tau_{n} / 2\right) !} & \text { if } \sigma_{n} \text { is even }\end{cases}
$$

and

$$
\mu_{p} \equiv-\lambda_{p} \frac{a^{(p-2) / 2}}{\lambda_{2}^{p / 2}}
$$


the integral takes the following form (owing to (8), (9), (12), (13)):

$$
J(a)=\sqrt{\frac{\pi}{\lambda_{2}}} \sum_{k_{3}, \ldots, k_{n}} A_{k_{3}, \ldots, k_{n}} \mu_{3}^{k_{3}} \cdots \mu_{n}^{k_{n}}
$$

Problems of convergence will be studied in Section 4.

This form, however, is not yet the most convenient one for our purpose, which is to prove the hypergeometric character of the series and to determine its region of convergence. Some notational difficulties will arise, which we try to avoid by the following conventions:

- Among the $k_{j}$, we denote by $\bar{k}_{j}$ those for which $j$ is an odd integer (of course, when $j$ is specified, as in $\bar{k}_{5}$, we simply write $k_{5}$ ).

$\circ \varepsilon_{j}= \begin{cases}0 & \text { if } j \text { is an even integer, } \\ 1 & \text { if } j \text { is an odd integer. }\end{cases}$

○ $l=E(n / 2)$ is the integer part of $n / 2$ (it is also the number of odd monomials in $\left.P_{n}(x)\right)$.

○ $\lambda$ is the greatest even integer $\leq l\left(\lambda\right.$ is just $\left.l-\varepsilon_{l}\right)$.

○ $E=\{3,5,7, \ldots, 2 l+1\}$.

$A_{k_{3}, \ldots, k_{n}}$ is nonzero only when $\sigma_{n}$ is even (this is the case when all the $\bar{k}_{j}$ are even numbers, or when an even number of $\bar{k}_{j}$ are odd). We can then split the summation in (14) and obtain $J(a)$ in a new form where the $\mu_{j}$ appear squared when $j$ is odd.

We set

$$
\begin{aligned}
& \alpha_{n}=\sum_{j=3}^{n}\left(1+\varepsilon_{j}\right) j k_{j}, \\
& \beta_{n}=\sum_{j=3}^{n}\left(1+\varepsilon_{j}\right)(j-2) k_{j}, \\
& \gamma_{n}=\frac{1}{\left(2 k_{3}\right) ! k_{4} ! \cdots\left[\left(1+\varepsilon_{n}\right) k_{n}\right] !}
\end{aligned}
$$

$\left(\alpha_{n}\right.$ and $\beta_{n}$ are, respectively, $\sigma_{n}\left(\bar{k}_{j} \rightarrow 2 \bar{k}_{j}\right)$ and $\tau_{n}\left(\bar{k}_{j} \rightarrow 2 \bar{k}_{j}\right)$; it is easy to see that they are even integers), and we note that

$$
\sigma_{n}\left(\bar{k}_{j} \rightarrow 2 \bar{k}_{j}+1\right)=\alpha_{n}+j, \quad \tau_{n}\left(\bar{k}_{j} \rightarrow 2 \bar{k}_{j}+1\right)=\beta_{n}+j-2
$$

Putting

$$
\mu_{3, \ldots, n}^{k_{3}, \ldots, n} \equiv\left(\mu_{3}^{2}\right)^{k_{3}} \mu_{4}^{k_{4}}\left(\mu_{5}^{2}\right)^{k_{5}} \cdots\left(\mu_{n}^{1+\varepsilon_{n}}\right)^{k_{n}}
$$


we finally obtain

$$
\begin{aligned}
& J(a)=\sqrt{\frac{\pi}{\lambda_{2}}}\left\{\sum_{k_{3}, \ldots, k_{n}} \gamma_{n} \frac{\Gamma\left(\alpha_{n} / 2+1 / 2\right)}{\left(\beta_{n} / 2\right) !} \mu_{3, \ldots, n}^{k_{3, \ldots, n}}\right. \\
& +\sum_{\substack{\text { pairs } \\
\left\{i_{1}, i_{2}\right\} \in E}} \mu_{i_{1}} \mu_{i_{2}} \sum_{k_{3}, \ldots, k_{n}} \frac{\gamma_{n}}{\left(2 \bar{k}_{i_{1}}-1\right)\left(2 \bar{k}_{i_{2}}+1\right)} \\
& \times \frac{\Gamma\left(\frac{\alpha_{n}+i_{1}+i_{2}}{2}+\frac{1}{2}\right)}{\left(\frac{\beta_{n}+i_{1}+i_{2}-4}{2}\right) !} \mu_{3, \ldots, n}^{k_{3}, \ldots, n} \\
& +\sum_{\substack{\text { quadruplets } \\
\left\{i_{1}, \ldots, i_{4}\right\} \in E}} \mu_{i_{1}} \cdots \mu_{i_{4}} \sum_{k_{3}, \ldots, k_{n}} \frac{\gamma_{n}}{\left(2 \bar{k}_{i_{1}}+1\right) \cdots\left(2 \bar{k}_{i_{4}}+1\right)} \\
& \times \frac{\Gamma\left(\frac{\alpha_{n}+i_{1}+\cdots+i_{4}}{2}+\frac{1}{2}\right)}{\left(\frac{\beta_{n}+i_{1}+\cdots+i_{4}-8}{2}\right) !} \mu_{3, \ldots, n}^{k_{3, \ldots, n}} \\
& +\cdots+\sum_{\substack{\lambda \text {-tuples } \\
\left\{i_{1}, \ldots, i_{\lambda}\right\} \in E}} \mu_{i_{1}} \cdots \mu_{i_{\lambda}} \sum_{k_{3, \ldots, n}} \frac{\gamma_{n}}{\left(2 \bar{k}_{i_{1}}+1\right) \cdots\left(2 \bar{k}_{i_{\lambda}}+1\right)} \\
& \left.\times \frac{\Gamma\left(\frac{\alpha_{n}+i_{1}+\cdots+i_{\lambda}}{2}+\frac{1}{2}\right)}{\left(\frac{\beta_{n}+i_{1}+\cdots+i_{\lambda}-2 \lambda}{2}\right) !} \mu_{3, \ldots, n}^{k_{3, \ldots, n}}\right\} .
\end{aligned}
$$

$J(a)$ is the sum of $1+C_{l}^{2}+C_{l}^{4}+\cdots+C_{l}^{\lambda}(n-2)$-tuple series in the variables $\mu_{3}^{2}, \mu_{4}, \mu_{5}^{2}, \ldots, \mu_{n}^{1+\varepsilon_{n}}$. ( $C_{l}^{k}$ are the binomial coefficients.)

We shall now show that these series are hypergeometric. A straightforward generalization of Horn's definition (see [6, p. 223] and [14, p. 53]) states that a series

$$
\sum_{m_{1}, \ldots, m_{n}} A_{m_{1}, \ldots, m_{n}} x_{1}^{m_{1}} \cdots x_{n}^{m_{n}}
$$

is an $n$-tuple hypergeometric series if the quotients

$$
f_{j}\left(m_{1}, \ldots, m_{n}\right)=\frac{A_{m_{1}, \ldots, m_{j}+1, \ldots, m_{n}}}{A_{m_{1}, \ldots, m_{j}, \ldots, m_{n}}}, \quad j \in\{1, \ldots, n\},
$$

are rational functions of the variables $m_{1}, \ldots, m_{n}$. The series is said to be homogeneous if numerator and denominator of $f_{j}$ have the same degree for every $j$.

It is easy, though rather tedious, to see that for each series in Eq. (19), numerator and denominator have the same degree: $j / 2$ if $j$ is an even integer, $j$ if $j$ is odd. Thus, $(19)$ is a sum of homogeneous $(n-2)$-tuple hypergeometric series.

3. Particular Cases. Upon using the Pochhammer symbol

$$
\frac{\Gamma(\alpha+k)}{\Gamma(\alpha)}=\alpha(\alpha+1) \cdots(\alpha+k-1) \equiv(\alpha)_{k}
$$

and the classical addition formula and Gauss's multiplication theorem [9]

$$
(\alpha)_{k+l}=(\alpha)_{k}(\alpha+k)_{l}
$$




$$
(\alpha)_{m k}=m^{m k} \prod_{j=1}^{m}\left(\frac{\alpha+j-1}{m}\right)_{k}
$$

the coefficients in $J(a)$ can be transformed to exhibit explicitly the hypergeometric nature of the series, at least in some particular cases.

(1) Single superquadratic term $P_{n}(x)=\lambda_{2} x^{2}+\lambda_{n} x^{n}$ [12], [4]:

$$
\left\{\begin{aligned}
J(a) & =\frac{\pi}{\sqrt{\lambda_{2}}}{ }_{n / 2} F_{n / 2-1}\left[\begin{array}{l}
\frac{1}{n}, \frac{3}{n}, \ldots, \frac{n-1}{n} \\
\frac{2}{n-2}, \frac{4}{n-2}, \ldots, \frac{n-2}{n-2} ; \nu_{n}
\end{array}\right] \quad \text { ( } n \text { even), } \\
& =\frac{\pi}{\sqrt{\lambda_{2}}}{ }_{n} F_{n-1}\left[\begin{array}{l}
\frac{1}{2 n}, \frac{3}{2 n}, \ldots, \frac{2 n-1}{2 n} \\
\frac{1}{n-2}, \frac{2}{n-2}, \ldots, \frac{n-2}{n-2}, \frac{1}{2}
\end{array} ; \nu_{n}^{2}\right.
\end{aligned} \quad \text { ( } n \text { odd }\right) .
$$

where

$$
\nu_{n}=\frac{(n / 2)^{n / 2}}{((n-2) / 2)^{(n-2) / 2}} \mu_{n}=-\frac{\lambda_{n}}{2}\left(\frac{n}{\lambda_{2}}\right)^{n / 2}\left(\frac{a}{n-2}\right)^{(n-2) / 2}
$$

and ${ }_{p} F_{q}$ is the Gaussian generalized hypergeometric series [6], [7], [14].

(2) Two even superquadratic terms $P_{n}(x)=\lambda_{2} x^{2}+\lambda_{m} x^{m}+\lambda_{n} x^{n}(m, n$ even integers):

$$
\begin{aligned}
& J(a)=\frac{\pi}{\sqrt{\lambda_{2}}} \\
& \times \sum_{k_{m}} n / 2 F_{n / 2-1}\left[\begin{array}{l}
\frac{1+m k_{m}}{n}, \frac{3+m k_{m}}{n}, \ldots, \frac{n-1+m k_{m}}{n} \\
\frac{2+(m-2) k_{m}}{n-2}, \frac{4+(m-2) k_{m}}{n-2}, \ldots, \frac{n-2+(m-2) k_{m}}{n-2} ; \nu_{n}
\end{array}\right] \\
& \times \frac{(1 / m)_{k_{m}}(3 / m)_{k_{m}} \cdots((m-1) / m)_{k_{m}}}{(2 /(m-2))_{k_{m}}(4 /(m-2))_{k_{m}} \cdots((m-2) /(m-2))_{k_{m}}} \frac{\nu_{m}^{k_{m}}}{k_{m} !} .
\end{aligned}
$$

The structure of the series (27) shows that it is a formal generalization of Appell series [6], [7], [14]. Note that in (27), because of the symmetry of $\sigma_{n}=m k_{m}+n k_{n}$, $m$ and $n$ having the same parity, we could exchange $m$ and $n$ and express $J(a)$ as a summation over the index $k_{n}$ with a "coefficient" ${ }_{m / 2} F_{m / 2-1}\left[\ldots(m \leftrightarrow n) ; \nu_{m}\right]$.

Although it might be possible, also in other cases, to show explicitly the hypergeometric structure with ${ }_{p} F_{q}$ coefficients, the general form (19) is still useful, especially in convergence problems. For two superquadratic terms, we have the following simplest cases:

$$
P_{4}(x)=\lambda_{2} x^{2}+\lambda_{3} x^{3}+\lambda_{4} x^{4}
$$

$$
J(a)=\sqrt{\frac{\pi}{\lambda_{2}}} \sum_{k_{3}, k_{4}} \frac{1}{\left(2 k_{3}\right) ! k_{4} !} \frac{\Gamma\left(3 k_{3}+2 k_{4}+\frac{1}{2}\right)}{\left(k_{3}+k_{4}\right) !}\left(\mu_{3}^{2}\right)^{k_{3}} \mu_{4}^{k_{4}}
$$




$$
\begin{aligned}
& P_{5}(x)=\lambda_{2} x^{2}+\lambda_{3} x^{3}+\lambda_{5} x^{5} \\
& J(a)=\sqrt{\frac{\pi}{\lambda_{2}}}\left[\sum_{k_{3}, k_{5}} \frac{1}{\left(2 k_{3}\right) !\left(2 k_{5}\right) !} \frac{\Gamma\left(3 k_{3}+5 k_{5}+\frac{1}{2}\right)}{\left(k_{3}+3 k_{5}\right) !}\left(\mu_{3}^{2}\right)^{k_{3}}\left(\mu_{5}^{2}\right)^{k_{5}}\right. \\
& \left.\quad+\mu_{3} \mu_{5} \sum_{k_{3}, k_{5}} \frac{1}{\left(2 k_{3}+1\right) !\left(2 k_{5}+1\right) !} \frac{\Gamma\left(3 k_{3}+5 k_{5}+\frac{9}{2}\right)}{\left(k_{3}+3 k_{5}+2\right) !}\left(\mu_{3}^{2}\right)^{k_{3}}\left(\mu_{5}^{2}\right)^{k_{5}}\right]
\end{aligned}
$$

$P_{6}(x)=\lambda_{2} x^{2}+\lambda_{4} x^{4}+\lambda_{6} x^{6} \quad($ which is (27) with $m=4$ and $n=6)$,

$$
J(a)=\sqrt{\frac{\pi}{\lambda_{2}}} \sum_{k_{4}, k_{6}} \frac{1}{k_{4} ! k_{6} !} \frac{\Gamma\left(2 k_{4}+3 k_{6}+\frac{1}{2}\right)}{\left(k_{4}+2 k_{6}\right) !} \mu_{4}^{k_{4}} \mu_{6}^{k_{6}} .
$$

Note that (29) is the simplest case where $J(a)$ appears like a sum of multiple hypergeometric series, the number of odd monomials in $P_{n}(x)$ being larger than one.

When the number of odd monomials in $P_{n}(x)$ is less than or equal to one, the summation reduces to a single hypergeometric series. Furthermore, every $\lambda_{j}$ which is zero reduces the multiplicity of the series.

4. Convergence Problems. For Gaussian simple hypergeometric series, such as (25), convergence is insured when $\left|\nu_{n}\right|<1$, but as soon as $P_{n}(x)$ involves more than one superquadratic term, we have to use Horn's theorem on convergence of multiple hypergeometric series [6], [7] and its generalization [14]. This theorem states that for general series like those occurring in (19), the associated radii of convergence (numbers $\xi_{j} \in \mathbf{R}^{+}$such that the series converges when $\left|x_{j}\right|<\xi_{j}$, $j \in\{1,2, \ldots, n\})$ lie in the "absolute space" $\left(\mathbf{R}^{+}\right)^{n}$ on a hypersurface $(\Sigma)$. When the series is homogeneous, $(\Sigma)$ may be defined as the hypersurface whose parametric equations are

$$
\xi_{j}=\frac{1}{\phi_{j}\left(m_{1}, \ldots, m_{n}\right)}
$$

with $\phi_{j}\left(m_{1}, \ldots, m_{n}\right)=\lim _{\eta \rightarrow \infty} f_{j}\left(m_{1} \eta, \ldots, m_{n} \eta\right)$ being finite for every $j$ because of homogeneity. (See Eq. (21).)

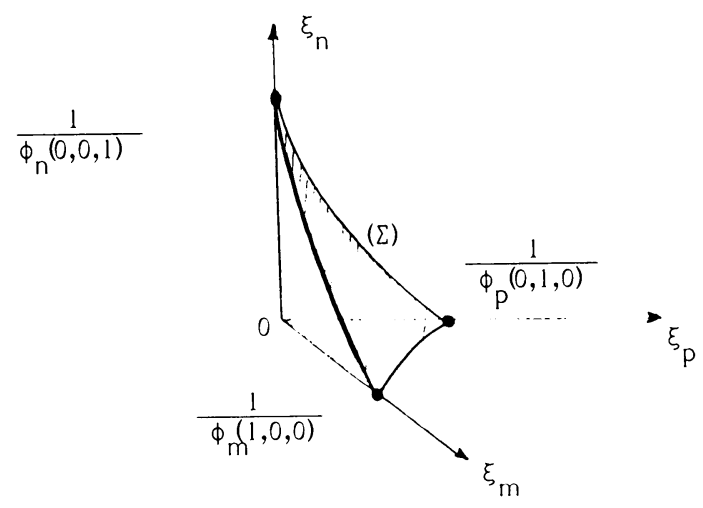

FIGURE 2 
Figure 2 shows $(\Sigma)$ in the case of three superquadratic terms $\lambda_{m} x^{m}, \lambda_{p} x^{p}$ and $\lambda_{n} x^{n}$ in $P_{n}(x)$.

Computation of $\xi_{j}$ in the general case is quite tedious, but it can be shown that every series occurring in (19) has the same $(\Sigma)$-hypersurface of convergence.

When there are only two superquadratic terms, $(\Sigma)$ reduces to a curve in $\left(\mathbf{R}^{+}\right)^{2}$, which we specify in the particular cases (28), (29) and (30). (The case (29) was already treated in [4].) The following table summarizes these results.

\begin{tabular}{|c|c|c|}
\hline particular series & $\begin{array}{l}\text { parametric equation } \\
\text { of the curve }(\Sigma)\end{array}$ & $\begin{array}{l}\text { region of convergence (S) } \\
\text { in }\left(\mathbb{R}^{+}\right)^{2} \text {. }\end{array}$ \\
\hline $\begin{array}{l}\text { (28) } \\
\text { converges when } \\
\left(\mu_{3}^{2},\left|\mu_{4}\right|\right) \\
\text { is in (S) }\end{array}$ & $\left\{\begin{array}{l}\xi_{3}=\frac{4(1+t)}{(3+2 t)^{3}} \\
\xi_{4}=\frac{t(1+t)}{(3+2 t)^{2}} \\
t=\frac{k_{4}}{k_{3}}\end{array}\right.$ & $\frac{1}{\xi_{4}}$ \\
\hline $\begin{array}{l}\text { (29) } \\
\text { converges when } \\
\left(\mu_{3}^{2}, \mu_{5}^{2}\right) \\
\text { is in (S) }\end{array}$ & $\left\{\begin{array}{l}\xi_{3}=\frac{4(1+3 t)}{(3+5 t)^{2}} \\
\xi_{5}=\frac{4 t^{2}(1+3 t)^{3}}{(3+5 t)^{5}} \\
t=\frac{k_{5}}{k_{3}}\end{array}\right.$ & $\frac{3125}{108}$ \\
\hline $\begin{array}{l}\text { (30) } \\
\text { converges when } \\
\left(\left|\mu_{4}\right|,\left|\mu_{6}\right|\right) \\
\text { is in (S) }\end{array}$ & $\left\{\begin{aligned} \xi_{4} & =\frac{1+2 t}{(2+3 t)^{2}} \\
\xi_{6} & =\frac{t(1+2 t)^{2}}{(2+3 t)^{3}} \\
t & =\frac{k_{6}}{k_{4}}\end{aligned}\right.$ & $\frac{4}{27}$ \\
\hline
\end{tabular}

It is easy to show that if we use the "natural" hypergeometric argument $\nu_{p}$ (defined in (26)) instead of $\mu_{p}$ in the $\left(\left|\nu_{p}\right|,\left|\nu_{q}\right|\right)$-plane $\left(\mathbf{R}^{+}\right)^{2},(\Sigma)$ always joins the extremities of the diagonal of the unit-square (or unit-hyperoctant in $\left(\mathbf{R}^{+}\right)^{n-2}$ ).

As a final remark we add that, probably, the series (14) (or (19)) may often be analytically continued, as is already the case for the simple example (25) when $n$ is even (see [12]; in this case, $J(a)$ may be defined for $\mu_{n}$ (or $\left.\nu_{n}\right) \rightarrow-\infty$.

Appendix: The Elliptic Case. When $P_{n}(x)$ involves a single superquadratic term of degree 3,4 , or 6 , we observed in the introduction that Eq. (2) can be 
transformed into a complete elliptic integral and straightforwardly integrated [12], [2], giving the following results ${ }_{2} F_{1}\left[\frac{1}{2}, \frac{1}{2}, 1 ; k^{2}\right] \equiv \frac{2}{\pi} K(k)$ where $K$ is the complete elliptic integral of the first kind):

$$
\begin{aligned}
& \int_{\alpha}^{\beta} \frac{d x}{\sqrt{a-\lambda_{2} x^{2}-\lambda_{3} x^{3}}}=\frac{\pi}{\sqrt{\lambda_{2}}}\left(1-k^{2}+k^{4}\right)^{1 / 4}{ }_{2} F_{1}\left[\frac{1}{2}, \frac{1}{2} ; 1 ; k^{2}\right],
\end{aligned}
$$

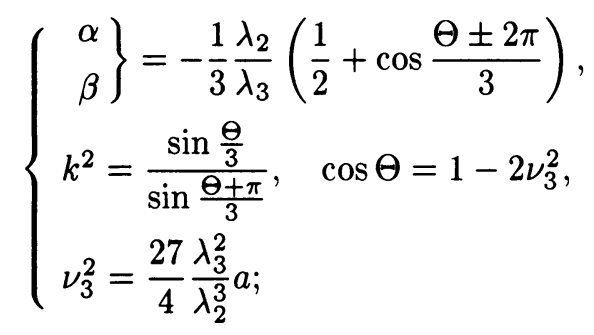

$$
\int_{\alpha}^{\beta} \frac{d x}{\sqrt{a-\lambda_{2} x^{2}-\lambda_{4} x^{4}}}
$$

$$
= \begin{cases}\frac{\pi}{\sqrt{\lambda_{2}}}\left(1-4 k^{2}+4 k^{4}\right)^{1 / 4}{ }_{2} F_{1}\left[\frac{1}{2}, \frac{1}{2} ; 1 ; k^{2}\right] & \text { if } \lambda_{4}>0, \\ \frac{\pi}{\sqrt{\lambda_{2}}}\left(1+\tilde{k}^{2}\right)^{1 / 2}{ }_{2} F_{1}\left[\frac{1}{2}, \frac{1}{2} ; 1 ; \tilde{k}^{2}\right] & \text { if } \lambda_{4}<0,\end{cases}
$$

$$
\left\{\begin{array}{l}
\beta=-\alpha=\frac{1}{2} \frac{\lambda_{2}}{\sqrt{\lambda_{4}}}\left[\left(1-\nu_{4}\right)^{1 / 2}-1\right]^{1 / 2} \quad \text { if } \lambda_{4}>0 \\
\beta=-\alpha=\frac{1}{2} \frac{\lambda_{2}}{\sqrt{-\lambda_{4}}}\left[1-\left(1-\nu_{4}\right)^{1 / 2}\right]^{1 / 2} \quad \text { if } \lambda_{4}<0 \\
k^{2}=\frac{1}{2}\left[1-\left(1-\nu_{4}\right)^{-1 / 2}\right], \quad \tilde{k}^{2}=\frac{1-\left(1-\nu_{4}\right)^{1 / 2}}{1+\left(1-\nu_{4}\right)^{1 / 2}} \\
\nu_{4}=-4 \frac{\lambda_{4}}{\lambda_{2}^{2}} a
\end{array}\right.
$$

$$
\int_{\alpha}^{\beta} \frac{d x}{\sqrt{a-\lambda_{2} x^{2}-\lambda_{6} x^{6}}}
$$

$$
= \begin{cases}\frac{\pi}{\sqrt{\lambda_{2}}}\left(1-16 k^{2}+16 k^{4}\right)^{1 / 4}{ }_{2} F_{1}\left[\frac{1}{2}, \frac{1}{2} ; 1 ; k^{2}\right] & \text { if } \lambda_{6}>0, \\ \frac{\pi}{\sqrt{\lambda_{2}}}\left(1-\tilde{k}^{2}+\tilde{k}^{4}\right)^{1 / 4}{ }_{2} F_{1}\left[\frac{1}{2}, \frac{1}{2} ; 1 ; k^{2}\right] & \text { if } \lambda_{6}<0,\end{cases}
$$

$$
\left\{\begin{array}{l}
\beta=-\alpha=\left\{\frac{1}{\sqrt{6}} \frac{\lambda_{2}}{\sqrt{\lambda_{6}}}\left(-\nu_{6}\right)^{1 / 6}\left[\left(1+\operatorname{th}^{-1} \Theta\right)^{1 / 3}+\left(1-\operatorname{th}^{-1} \Theta\right)^{1 / 3}\right]\right\}^{1 / 2} \text { if } \lambda_{6}>0 \\
\beta=-\alpha=\left[\frac{2}{\sqrt{6}} \frac{\lambda_{2}}{\sqrt{-\lambda_{6}}} \cos \frac{\tilde{\Theta}-2 \pi}{3}\right]^{1 / 2} \quad \text { if } \lambda_{6}<0 \\
\operatorname{sh} \Theta=\sqrt{-\nu_{6}}, \quad \cos \tilde{\Theta}=-\sqrt{\nu_{6}}, \\
\tilde{k}^{2}=\frac{\sin \frac{2 \tilde{\Theta}-\pi}{3}}{\sin \frac{2 \tilde{\Theta}}{3}}, \frac{\left(1-2 k^{2}\right)\left(1+32 k^{2}-32 k^{4}\right)}{\left(1-16 k^{2}+16 k^{4}\right)^{3 / 2}}=\frac{\left(1-2 \tilde{k}^{2}\right)\left(1+\frac{1}{2} \tilde{k}^{2}-\frac{1}{2} \tilde{k}^{4}\right)}{\left(1-\tilde{k}^{2}+\tilde{k}^{4}\right)^{3 / 2}} \\
\nu_{6}=-\frac{27}{4} \frac{\lambda_{6}}{\lambda_{2}^{3}} a^{2}
\end{array}\right.
$$


(Formulae equivalent to (34) are given by Lakshmanan and Prabhakaran [11]. Equation (25) of the present paper gives much simpler results. The values of $\alpha$ and $\beta$ in "hypergeometric form" are derived from formulae [12] obtained by the Lagrange-Bürmann Theorem; see also [5].)

$$
\begin{aligned}
& \int_{\alpha}^{\beta} \frac{d x}{\sqrt{a-\lambda_{2} x^{2}-\lambda_{3} x^{3}}}=\frac{\pi}{\sqrt{\lambda_{2}}}{ }_{2} F_{1}\left[\frac{1}{6}, \frac{5}{6} ; 1 ; \frac{27}{4} \frac{\lambda_{3}^{2}}{\lambda_{2}^{3}} a\right], \\
& \left.\begin{array}{l}
\alpha \\
\beta
\end{array}\right\}= \pm \sqrt{\frac{a}{\lambda_{2}}}{ }_{2} F_{1}\left[\frac{1}{6}, \frac{5}{6} ; \frac{3}{2} ; \frac{27}{4} \frac{\lambda_{3}^{2}}{\lambda_{2}} a\right]-\frac{\lambda_{3} a}{\lambda_{2}^{2}}{ }_{2} F_{1}\left[\frac{2}{3}, \frac{4}{3} ; \frac{3}{2} ; \frac{27}{4} \frac{\lambda_{3}^{2}}{\lambda_{2}} a\right] \text {; } \\
& \int_{\alpha}^{\beta} \frac{d x}{\sqrt{a-\lambda_{2} x^{2}-\lambda_{4} x^{4}}}=\frac{\pi}{\sqrt{\lambda_{2}}}{ }_{2} F_{1}\left[\frac{1}{4}, \frac{3}{4} ; 1 ;-4 \frac{\lambda_{4}}{\lambda_{2}^{2}} a\right] \text {, } \\
& \beta=-\alpha=\sqrt{\frac{a}{\lambda_{2}}}{ }_{2} F_{1}\left[\frac{1}{4}, \frac{1}{4} ; \frac{3}{2} ;-4 \frac{\lambda_{4}}{\lambda_{2}^{2}} a\right] \text {; } \\
& \int_{\alpha}^{\beta} \frac{d x}{\sqrt{a-\lambda_{2} x^{2}-\lambda_{6} x^{6}}}=\frac{\pi}{\sqrt{\lambda_{2}}}{ }_{2} F_{1}\left[\frac{1}{6}, \frac{5}{6} ; 1 ;-\frac{27}{4} \frac{\lambda_{6}}{\lambda_{2}^{3}} a^{2}\right], \\
& \beta=-\alpha=\sqrt{\frac{a}{\lambda_{2}}}{ }_{3} F_{2}\left[\begin{array}{c}
\frac{1}{6}, \frac{1}{2}, \frac{5}{6} \\
\frac{3}{2}, \frac{5}{4}
\end{array} ;-\frac{27}{4} \frac{\lambda_{6}}{\lambda_{2}^{3}} a^{2}\right] .
\end{aligned}
$$

(Identification of (35), (36), (37), respectively, with (32), (33), (34) is carried out in [12], using transformation relations for Gaussian hypergeometric functions established by Goursat [9]. Integrals (35) to (37) may also be identified with Legendre functions [2].)

$$
\begin{aligned}
& \int_{\alpha}^{\beta} \frac{d x}{\sqrt{a-\lambda_{2} x^{2}-\lambda_{3} x^{3}}}=\frac{\pi}{\sqrt{\lambda_{2}}} P_{-1 / 6}\left(1-\frac{27}{2} \frac{\lambda_{3}^{2}}{\left.\lambda_{2}^{3} a\right)}\right. \\
& \int_{\alpha}^{\beta} \frac{d x}{\sqrt{a-\lambda_{2} x^{2}-\lambda_{4} x^{4}}}=\frac{\pi}{\sqrt{\lambda_{2}}} P_{-1 / 4}\left(1+8 \frac{\lambda_{4}}{\lambda_{2}^{2}} a\right) \\
& \int_{\alpha}^{\beta} \frac{d x}{\sqrt{a-\lambda_{2} x^{2}-\lambda_{6} x^{6}}}=\frac{\pi}{\sqrt{\lambda_{2}}} P_{-1 / 6}\left(1+\frac{27}{2} \frac{\lambda_{6}}{\lambda_{3}^{2}} a^{2}\right)
\end{aligned}
$$

where $P_{\sigma}(x)$ is the solution of Legendre's differential equation

$$
\left(1-x^{2}\right) \frac{d^{2} f}{d x^{2}}-2 x \frac{d f}{d x}+\sigma(\sigma+1) f=0
$$

Laboratoire de Physique Appliquée

Faculté des Sciences

Université de Pau et des Pays de l'Adour

64000 Pau, France

1. R CABoz, J. P. Codaccioni \& F. Constantinescu, "Taylor series for the Dirac function on perturbed surfaces with applications to mechanics," Math. Methods Appl. Sci., v. 7, 1985, pp. 416-425.

2. R. CABOZ \& J. F. LOISEAU, "Lien entre période et hauteur normalisée à l'intérieur du puits de potentiel pour l'oscillateur anharmonique," C.R. Acad. Sci. Paris, v. 296, 1983, pp. 1753-1756. 
3. B. C. CARLson, Special Functions of Applied Mathematics, Academic Press, New York, 1977.

4. J. P. CODACCIONI \& R. CABOz, "Anharmonic oscillators and generalized hypergeometric functions," J. Math. Phys., v. 25, 1984, pp. 2436-2438.

5. J. P. CODACCIONI \& R. CABOZ, "Anharmonic oscillators revisited," Internat. J. Non-Linear Mech., v. 20, 1985, pp. 291-295.

6. A. ERdÉLYI, Higher Transcendental Functions, Vol. 1 (Bateman Manuscript Project), McGraw-Hill, New York, 1953.

7. H. ExTON, Multiple Hypergeometric Functions and Applications, Ellis Horwood, Chichester, 1976.

8. I. M. Gelfand \& G. E. Shilov, Generalized Functions, Academic Press, New York, 1964.

9. E. GOURSAT, "Sur l'équation différentielle linéaire qui admet pour intégrale la série hypergéometrique," Ann. Ecole Norm. Sup., v. 10, suppl. Paris, 1881.

10. J. S. Gradshteyn \& I. M. RYzhiK, Table of Integrals, Series and Products, Academic Press, New York, 1980.

11. M. LAKShMANAN \& J. PrabhaKARAN, "The energy level of an $x^{6}$ anharmonic oscillator," Lett. Nuovo Cimento, v. 7, 1973, pp. 689-692.

12. J. F. LoIseau, Contribution à l'Étude des Solutions Exactes et Approchées de l'Oscillateur Anharmonique Monômial en Mécanique Classique (Thèse d'Etat), Université de Pau et des Pays de l'Adour, 1986.

13. M. S. SPIEgel, Formules et Tables de Mathématiques (Série Schaum), McGraw-Hill, New York, 1983.

14. H. M. SRIVAstaVA \& P. W. KARLSSON, Multiple Gaussian Hypergeometric Series, Ellis Horwood, Chichester, 1985.

Reference [14] contains a very complete and up-to-date bibliography on Multiple Hypergeometric Series and Functions. 